\title{
Revealing New Atomic-scale Information about Materials by Improving the Quality and Quantifiability of Aberration-corrected STEM Data
}

\author{
Andrew B. Yankovich ${ }^{1}$, Jie Feng ${ }^{1}$, Alex Kvit ${ }^{1}$, Thomas Slater ${ }^{2}$, Sarah Haigh $^{2}$, Dane Morgan ${ }^{1}$, and Paul \\ M. Voyles ${ }^{1}$ \\ ${ }^{1}$ Dept. of Materials Science and Engineering, University of Wisconsin, Madison, WI 53706, USA. \\ ${ }^{2}$ School of Materials, University of Manchester, Manchester, M13 9PL, UK.
}

Recent advances in aberration-corrected scanning transmission electron microscopy (AC-STEM) have shown the world previously unattainable views into the atomic structure and composition of materials. Thanks to improved optics, experimental and environmental factors often limit the quality of information accessed by AC-STEM. However, collecting and processing AC-STEM data using new techniques from data science can help overcome these limitations, opening the door to new atomic-scale materials information by improving our ability to determine atomic column positions, measure 3D structures, detect single point defects, and determine the atomic-scale composition of materials.

Typically for identifying atomic column positions in STEM images, practical limits are encountered before the fundamental signal to noise ratio (SNR) limit. The most common practical limit is distortions caused by instabilities during image acquisition. Recently, we developed a non-rigid registration (NRR) technique that overcomes this practical limit by correcting all types of image distortions present in STEM images [1][2]. Other methods have also been developed to address this issue [3]. The NRR and averaging of high angle annular dark field (HAADF) STEM image series greatly increases the image SNR and allows for sub-pm precision of beam insensitive single crystal materials. NRR and averaging HAADF STEM image series of Pt nanocatalysts have allowed measurements of pm-scale surface atom bond length variation that may help explain their catalytic activity. The atomic structure of a $\mathrm{Au}$ nanoparticle was measured with 1-2 pm precision using NRR at $1 \%$ of the dose of the Pt results [4], showing the high-precision NRR technique can be used on more beam sensitive material systems.

Extracting 3D information from 2D TEM/STEM images is a long-standing problem in electron microscopy. Standardless atom counting allows for 3D atomic structure information to be measured from HAADF STEM and HRTEM images by comparing experimental and simulated image intensities [5][6]. Previously, the uncertainty in this measurement was limited by image Poisson noise. NRR and averaging allows for standardless atom counting with the uncertainty no longer dominated by Poisson noise [2]. The unique determination of the number of atoms in atomic columns is now possible, although not yet demonstrated due to other experimental limitations, such as sample preparation artifacts.

Point defects are critical to the properties of a wide range of materials, including semiconductors and solid oxides used in fuel cells. Imaging substitutional and interstitial dopant impurity atoms is possible using HAADF STEM [7], as well as determining the depth of the dopant in the TEM specimen [8]. However, experimentally imaging single atomic vacancies remains a challenge. We have conducted extensive frozen phonon multislice simulations that predict the visibility of $\mathrm{La}$ vacancies in $\mathrm{LaMnO}_{3}$, both by the reduced atomic column intensity and by atomic column position distortions around the vacancy. NRR and averaging HAADF STEM images of $\mathrm{LaMnO}_{3}$ improves the SNR and the image precision sufficiently to potentially experimentally detect single La vacancies. Figure 1(a) shows a NRR and averaged HAADF STEM image of a $\mathrm{LaMnO}_{3}$ film. Figure 1(b) shows the visibility of each atomic 
column relative to its nearest 8 atomic columns. The atom column in (b) marked by the green box has a $\sim 7 \%$ visibility, matching simulated predictions of single La vacancies, making it a candidate single vacancy.

Atomic-scale composition can be determined using STEM EDS spectrum imaging (SI), however due to a low efficiency of producing and collecting x-ray signals, long total dwell times that introduce spatial distortions are required. Currently, to minimize the distortions, multiple SIs are acquired using online drift-correction software, then summed, while the individual SIs and simultaneous HAADF images are discarded. Figure 2 shows that the quality of EDS SIs is improved by saving the simultaneously acquired raw HAADF and EDS SI series of $\mathrm{Nd}_{2 / 3} \mathrm{TiO}_{3}$ [9] and applying NRR. Figure 2 shows $\mathrm{Nd}$ and Ti elemental maps extracted from a NRR SI, standard drift correction SI, and a single long-dwell time SI. NRR of the EDS SI produces elemental maps with less spatial distortions, more atomic localization of x-ray signals, and higher contrast. Quantitative comparison between these techniques and additional methods of denoising EDS SIs will be discussed. [10]

\section{References:}

[1] Berkels et al. Ultramicroscopy, 138, 46 (2013).

[2] Yankovich et al. Nature Communications, 5, 4155 (2014).

[3] Sang et al. Ultramicroscopy, 138, 28-35 (2014).

[4] Yankovich et al. Advanced Structural and Chemical

Imaging, accepted

(2015).

[5] LeBeau et al. Nano

Letters, 10, 4405

(2010).

[6] Jia et al. Nature

Materials, 13, 1044

(2014).

[7] Voyles et

al. Nature, 416, 826

(2002).

[8] Hwang et al. PRL, 111, no. 26, 266101

(2013).

[9] Azough et al.

Chemistry of Materials, 27, 2, 497 (2015).

[10] Work was funded by the Department of Energy, Basic Energy Sciences (DE-FG0208ER46547).

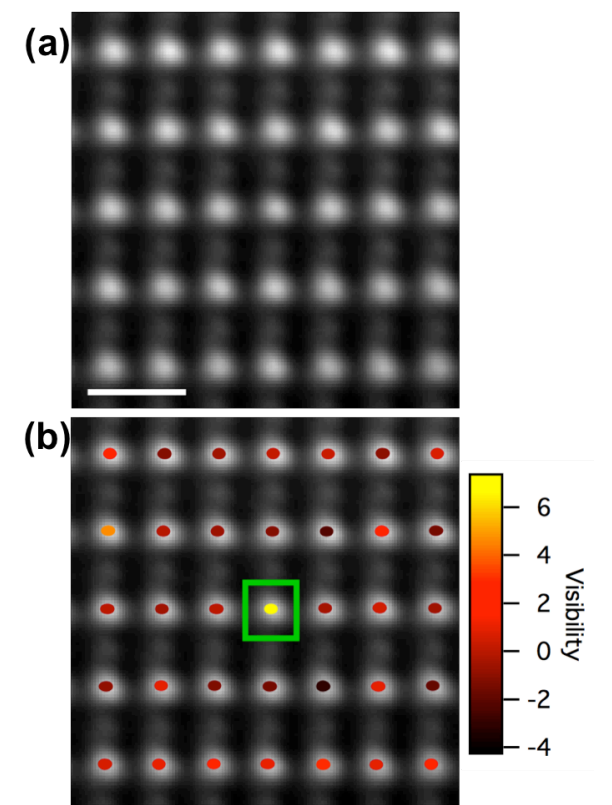

Figure 1: (a) NRR and averaged HAADF STEM image of [100] $\mathrm{LaMnO}_{3}$ grown on a $\mathrm{DyScO}_{3}$. Scale bar is $0.5 \mathrm{~nm}$. (b) Visibility map of each atomic column in (a).

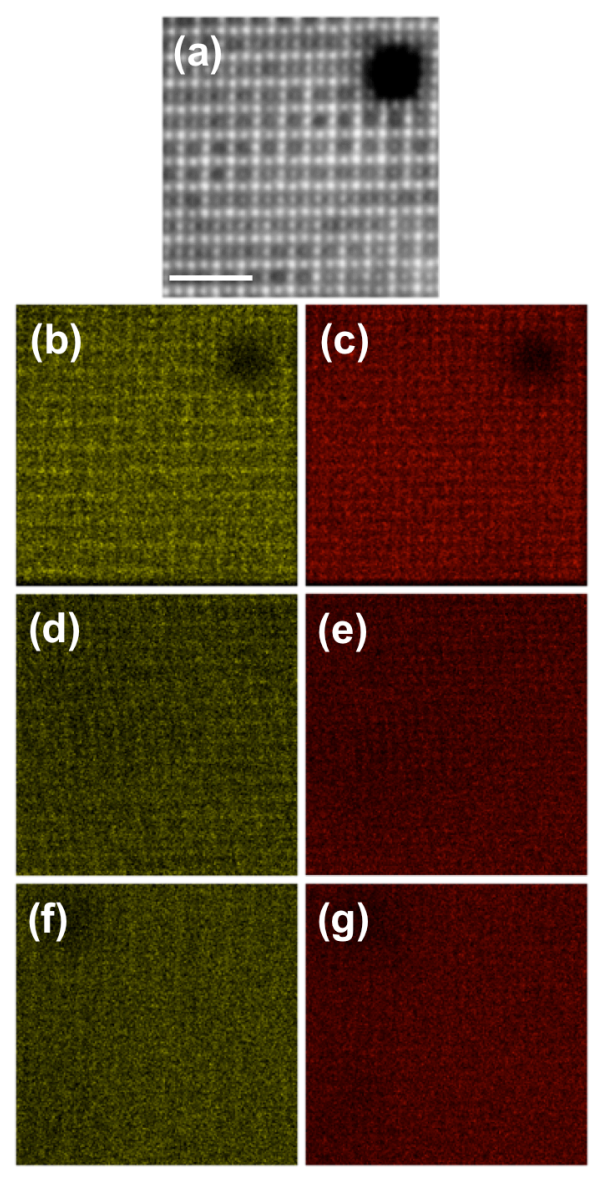

Figure 2: (a) NRR and averaged HAADF STEM image of $\mathrm{Nd}_{2 / 3} \mathrm{TiO}_{3}$. (b)-(g) EDS elemental maps using the $\mathrm{Nd} \mathrm{L} \alpha$ peak (b)(d)(f) and the $\mathrm{Ti}$ $\mathrm{K} \alpha$ peak (c)(e)(g). (b)\&(c) Maps from a sum of 98 SIs after NRR. (d)\&(e) Maps from a single long exposure SI. (f)\&(g) Maps from a sum of 100 drift-corrected SIs. The total counts in each of the 3 SI methods are approximately constant. No images have been smoothed. Scale bar is $2.5 \mathrm{~nm}$. 